\title{
Clinical characteristics of a group of deaths with COVID-19 pneumonia in Wuhan, China: a retrospective case series
}

Tao Yao ${ }^{1,2+}$, Yan Gao ${ }^{1,2+}$, Qin Cui ${ }^{1,2+}$, Bo Peng ${ }^{1,2}$, Yan Chen ${ }^{1,2}$, Jiansheng $\mathrm{Li}^{1,2}$, Chao Huang ${ }^{1,3}$, Chunping He ${ }^{1,3}$, Jie Pu ${ }^{1,2}$, Jiajun Wei ${ }^{1,2}$, Yanqiang Zhan ${ }^{1,2}$, Jie Yan ${ }^{1,4}$, Jinghua Tian ${ }^{1,5}$, Zhaohui Zhang ${ }^{1,2^{*}}$ and Zhichao Liu ${ }^{1,2^{*}}$ (D)

\begin{abstract}
Background: With the widespread outbreak of novel coronavirus diseases 2019(COVID-19), more and more death cases were reported, however, limited data are available for the patients who died. We aimed to explore the clinical characteristics of deaths with COVID-19 pneumonia.

Methods: We abstracted and analyzed epidemiological, demographic, clinical, and laboratory data from 83 death cases with COVID-19 pneumonia in East Hospital of Wuhan University Renmin Hospital, between January 26, 2020, and February 28, 2020.

Results: Of the 83 deaths, none was the medical staff. The mean age was 71.8 years (SD 13.2; range, 34-97 years) and 53(63.9\%) were male. The median from onset to admission was 10 days (IQR 7-14: range, 2-43 days), to death was 17 days (IQR 14-21: range, 6-54 days). Most deaths (66[80\%]) had underlying comorbid diseases, the most of which was hypertension [47(57\%)]. The main initial symptoms of these 83 deaths were shortness of breath(98.8\%), fever(94\%), and myalgia or fatigue(90.4\%). Laboratory analyses showed the lymphocytopenia in 69(83\%) deaths, hypoalbuminemia in $77(93 \%)$ deaths, the elevation of lactate dehydrogenase in 79(95\%) deaths, procalcitonin in 69(83\%) deaths and C-reactive protein in 79(95\%) deaths. All 83 patients received antiviral treatment, 81(97.6\%) deaths received antibiotic therapy, 54(65.1\%) deaths received glucocorticoid therapy, and 20(24.1\%) patients received invasive mechanical ventilation.
\end{abstract}

Conclusion: Most of the deaths with COVID-19 pneumonia were elderly patients with underlying comorbid diseases, especially those over 70 years of age. The time of death after the onset of the disease was mostly 15-21 days. More care should be given to the elderly in further prevention and control strategies of COVID-19.

Keywords: Characteristics, COVID-19, Pneumonia, Death

\footnotetext{
*Correspondence: zhangzhaohui2020@126.com; zhichao.liu@whu.edu.cn

${ }^{\dagger}$ Tao Yao, Yan Gao and Qin Cui contributed equally to this work.

'Union Department of Infection Disease, Wuhan University Renmin Hospital, Wuhan 430060, China

Full list of author information is available at the end of the article
}

(c) The Author(s). 2020 Open Access This article is licensed under a Creative Commons Attribution 4.0 International License, which permits use, sharing, adaptation, distribution and reproduction in any medium or format, as long as you give appropriate credit to the original author(s) and the source, provide a link to the Creative Commons licence, and indicate if changes were made. The images or other third party material in this article are included in the article's Creative Commons licence, unless indicated otherwise in a credit line to the material. If material is not included in the article's Creative Commons licence and your intended use is not permitted by statutory regulation or exceeds the permitted use, you will need to obtain permission directly from the copyright holder. To view a copy of this licence, visit http://creativecommons.org/licenses/by/4.0/ The Creative Commons Public Domain Dedication waiver (http://creativecommons.org/publicdomain/zero/1.0/) applies to the data made available in this article, unless otherwise stated in a credit line to the data. 


\section{Background}

The novel coronavirus diseases 2019(COVID-19) first reported in Wuhan, Hubei province, China $[1,2]$. It then spread widely in China and other nations around the world [3, 4]. With the COVID-19 global pandemic, more and more cases of death were reported [5-7]. As of July $1,2020,10,358,119$ cases of COVID-19 have been confirmed and 508,085 of them died according to WHO Coronavirus Disease (COVID-19) Dashboard [8].

The previous studies have already reported the features of severe cases and death cases of COVID-19 [3, 9-11]. The result of a study from Wuhan in China showed that compared with survivors with COVID-19 pneumonia, non-survivors were older, more likely to develop ARDS, and more likely to receive mechanical ventilation, either invasively or non-invasively [9]. A survey from New York City in the United States found that older age, chronic cardiac disease, chronic pulmonary disease, higher concentrations of interleukin-6, and higher concentrations of D-dimer were independently associated with in-hospital mortality $[10,11]$. As the number of patients confirmed COVID-19 and deaths continue to increase worldwide, reduce patient mortality, and improve prognosis has become an emergent issue confronting the current epidemic situation $[8,10,11]$. Further studies on the clinical features of death cases are needed, which could be valuable for the early specific management of critical patients.

In this study, we retrospectively collected and described detailed epidemiological, demographic, clinical, and laboratory characteristics of 83 deaths with COVID19 pneumonia who had been admitted to East Hospital Wuhan University Renmin Hospital, which was one of the first designated hospitals in Wuhan to admit severe patients with COVID-19.

\section{Methods}

\section{Study design and patients}

This is a single-center retrospective study. We reviewed all patients with COVID-19 pneumonia who were admitted to East Hospital of Wuhan University Renmin Hospital as of February 28, 2020, and collected data on death cases in hospital. East Hospital of Wuhan University Renmin Hospital located in Wuhan, Hubei Province, China, was designated as one of the first hospitals to admit severe adult patients with COVID-19 pneumonia by government. The diagnostic standard of COVID-19 pneumonia is based on the 4th edition Protocol of Novel coronavirus pneumonia Prevention and Control Program issued by the National Health Commission of the People's Republic of China [12].

This study protocol complied with the Medical Ethical Committee of Wuhan University Renmin Hospital (No.WDYR2020-k050). Written informed consent was waived due to the rapid emergence of this infectious disease.

\section{Data collection}

Several investigators reviewed the electronic medical record system of the hospital, and abstracted epidemiological, demographic, clinical, and laboratory data from death cases with COVID-19 pneumonia as of February 28, 2020. The other two researchers reviewed and checked the data collected. The investigators directly contacted their families to refine the data if some epidemiological data of patients were not available in the medical record.

Nasopharyngeal swabs were obtained from all patients at admission. All samples were processed at the Department of Clinical Laboratory of Wuhan University Renmin Hospital. COVID-19 was confirmed by realtime polymerase chain reaction testing according to WHO guidelines for laboratory testing [13]. Positive confirmed patients with COVID-19 infection were defined as at least 2 positive test results and the detection interval should be at least $24 \mathrm{~h}$.

\section{Statistical analysis}

Continuous variables are expressed as the means \pm standard deviations (SD) if they are normally distributed or medians (interquartile ranges, IQR) if they are not. Categorical variables are expressed as frequencies and percentages. All statistical analysis was performed with SPSS, version 25.0 (SPSS Inc., Chicago, IL, USA).

\section{Results}

As of February 28, 2020, 83(5.7\%) of the 1439 patients with COVID-19 pneumonia admitted to the hospital, died in hospital. None of the 83 deaths were medical staff, and there was no definite exposure history of patients with suspected or confirmed COVID-19.

The mean age was 71.8 years (SD 13.2; range, 34-97 years), including 26 patients over 80 years (31\%) and 2 patient under 40 years (2\%). Among the deaths, $53(63.9 \%)$ were male. The initial symptoms of these 83 patients were shortness of breath(98.8\%), fever(94\%), myalgia or fatigue(90.4\%), anorexia(82\%), cough(60.2\%), hemoptysis(6\%), pharyngalgia(6\%), headache(3.6\%), nausea or vomiting $(2.4 \%)$ and diarrhea(4.8\%) (Table 1$)$.

Onset-to-admission interval of the 83 deaths was between 2 and 43 days (median 10 days, IQR 7-14), most of them were 6-10 days (43\%). Onset-to-death interval was between 6 and 54 days (median 17 days, IQR 1421 ), and most of them were 15-21 days for $50 \%$ of women and $45 \%$ of men (Table 2). Figure 1 showed the date distribution of illness onset in all 83 patients. As described in this figure, the most dates of illness onset are between January 20 and January 28, 2020. 
Table 1 Demographics and clinical characteristics of 83 deaths with COVID-19 pneumonia

\begin{tabular}{ll}
\hline Characteristics & Patients, $\mathbf{n}(\%)$ \\
\hline Demographic factors & \\
Age(years) Mean (SD), & $71.8(13.2)$ \\
$<40$ & $2(2 \%)$ \\
$40-49$ & $3(4 \%)$ \\
$50-59$ & $10(12 \%)$ \\
$60-69$ & $15(18 \%)$ \\
$70-79$ & $27(33 \%)$ \\
$\geq 80$ & $26(31 \%)$ \\
Sex & \\
Men & $53(63.9 \%)$ \\
Women & $30(36.1 \%)$ \\
Clinical Characteristics & \\
Signs and symptoms at onset & \\
Fever & \\
Myalgia or fatigue & $78(94 \%)$ \\
Cough & $75(90.4 \%)$ \\
Pharyngalgia & $50(60.2 \%)$ \\
Headache & $5(6.0 \%)$ \\
Haemoptysis & $3(3.6 \%)$ \\
Anortness of breath & $5(6.0 \%)$ \\
Nausea or Vomiting & $81(98.8 \%)$ \\
\hline & $70(84.3 \%)$ \\
Diarrhoea & $2(2.4 \%)$ \\
\hline & $4(4.8 \%)$ \\
\hline &
\end{tabular}

Data are $\mathrm{n}(\%)$ or mean (SD)

Of the 83 deaths, 66 patients $(80 \%)$ had chronic comorbidities, the most of which was hypertension(57\%), followed by cardiovascular disease(31\%), diabetes (26\%), cerebrovascular disease(17\%), chronic lung disease(19.3\%), chronic renal disease (6\%), malignancy $(6 \%)$ and chronic liver disease (4\%) (Table 3).

The main laboratory findings of the deaths on admission were shown in Table 4. The results of the blood count showed that white blood cell count in 5(6\%) patients, lymphocyte count in 69(83\%) patients, hemoglobin in 34(41\%) patients, and platelets in 24(29\%) patients were below the normal range. Also, white blood cell count in $34(41 \%)$ patients and mononuclear leucocyte in $16(19 \%)$ patients were above the normal range.

On admission, many patients had an abnormal liver function and renal function. Aspartate aminotransferase in $57 \%$ patients, gamma-glutamyl transpeptidase in $49 \%$ patients, serum creatinine in $47 \%$ patients, and blood urea nitrogen in 53\% patients were above the normal range. Albumin levels were lower than normal in $93 \%$ patients. Lactate dehydrogenase increased in $95 \%$ patients. Most patients have abnormal coagulation
Table 2 Key Epidemiologic Variables of 83 deaths with COVID19 pneumonia

\begin{tabular}{ll}
\hline Variable & Patients, $\mathbf{n}(\%)$ \\
\hline Onset-to-admission interval & $10(7-14)$ \\
$1-5 d$ & $11(13 \%)$ \\
$6-10 d$ & $36(43 \%)$ \\
$10-15 d$ & $25(30 \%)$ \\
$\geq 16 d$ & $11(13 \%)$ \\
Onset-to-death interval & $17(14-21)$ \\
Women & $16(14-20)$ \\
$1-7 d$ & $1(3 \%)^{a}$ \\
$8-14 d$ & $8(27 \%)^{a}$ \\
$15-21 d$ & $15(50 \%)^{a}$ \\
$22-28 d$ & $4(13 \%)^{a}$ \\
$\geq 29 d$ & $2(7 \%)^{a}$ \\
Men & $18(14-23)$ \\
$1-7 d$ & $2(4 \%)^{b}$ \\
$8-14 d$ & $13(25 \%)^{b}$ \\
$15-21 d$ & $24(45 \%)^{b}$ \\
$22-28 d$ & $11(21 \%)^{b}$ \\
$\geq 29 d$ & $3(6 \%)^{b}$ \\
\hline
\end{tabular}

Data are median (IQR) or $\mathrm{n}(\%) .{ }^{\mathrm{a}}$ Proportion of women; ${ }^{\mathrm{b}}$ Proportion of men

function, which showed the elevation of D-dimer in $94 \%$ patients, the extension of Prothrombin time in 43\% patients, and Activated partial thromboplastin time in 33\% patients. Moreover, procalcitonin (83\%) and C-reactive protein $(95 \%)$ increased above the normal range in most patients.

Each patient performed a chest CT scan on admission, and pneumonia was confirmed in all 83 patients, and 71 patients were involved in the bilateral lung (Table 5). Radiographic features from chest CT scans mainly included ground glass opacity, consolidation, air bronchogram, bronchial dilatation, and pleural effusion or thickening. Multiple patchy ground-glass shadows were the main feature in the chest CT of most patients, followed by consolidation (Table 5; Fig. 2).

The main complications were acute respiratory failure $(85.5 \%)$, sepsis $(83.1 \%)$, heart failure(44.6\%), acute kidney injury (26.5\%), gastrointestinal bleeding(19.2\%), acute liver injury(10.8\%), acute myocardial infarction(7.2\%) (Table 6) In drug treatment, all 83 patients received monotherapy and combination antiviral therapy for 5-10 days, and all of them received Abidole, 58 received Oseltamivir, 16 received Ribavirin, 4 received Lopinavir and Ritonavir. Among them, 57 (68.7\%)patients received Abidole and Oseltamivir, 16(19.3\%) received Abidole and Ribavirin, 3(3.6\%) received Abidole and Lopinavir and Ritonavir, and 1(1.2\%) received Lopinavir, Oseltamivir and Lopinavir and Ritonavir. Of 83 


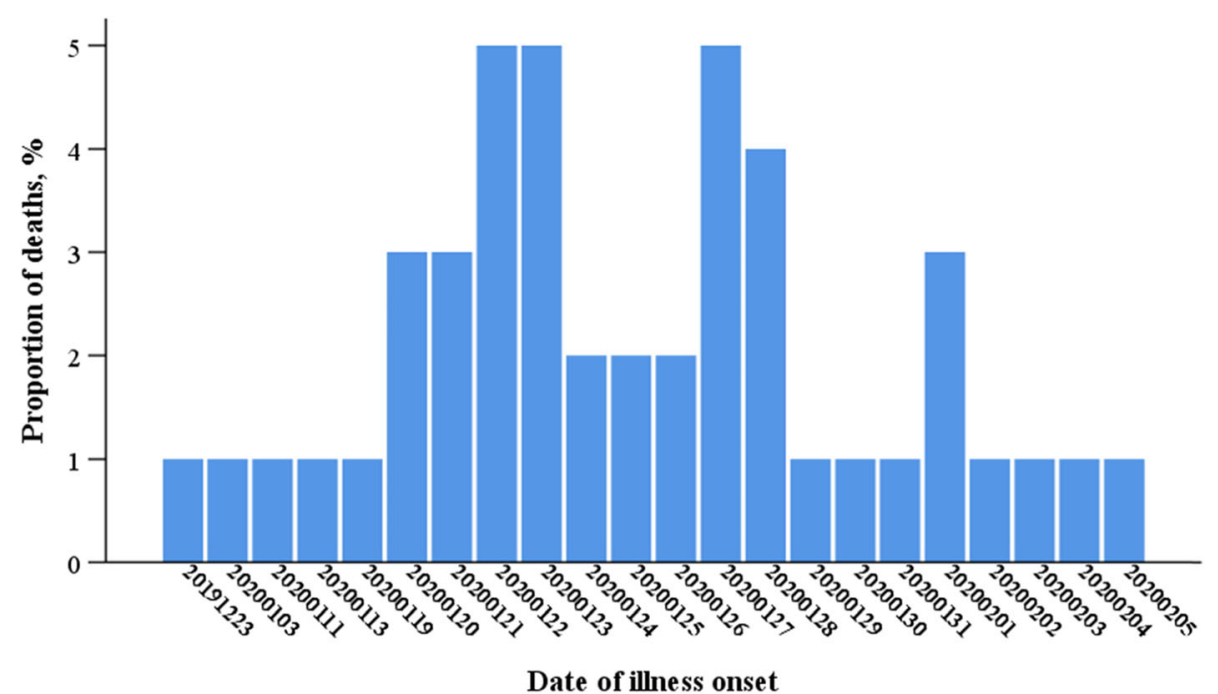

Fig. 1 Date of illness onset of 83 deaths with COVID-19 pneumonia

patients, $81(97.6 \%)$ patients received antibiotics therapy including Penicillins(28.9\%), Cephalosporins(62.7\%), Quinolone(42.4\%) and Vancomycin(13.3\%). 54(65.1\%) patients received glucocorticoid therapy and 39(47.0\%) patients received intravenous immunoglobulin therapy. In addition to drug treatment, all patients received oxygen therapy, 20 of them(24.1\%) received invasive mechanical ventilation(IMV), 51 of them(61.4\%) received non-invasive mechanical ventilation(NIMV), and 12 of them $(14.5 \%)$ received high flow nasal cannula. 6(7.3\%) patients received continuous replacement

Table 3 Characteristics of comorbidity of 83 deaths with COVID-19 pneumonia

\begin{tabular}{ll}
\hline & Patients, $\mathbf{n}(\%)$ \\
\hline Comorbidities & $66(80 \%)$ \\
Hypertension & $47(57 \%)$ \\
Diabetes & $14(26 \%)$ \\
Cardiovascular & $26(31 \%)$ \\
Cerebrovascular disease & $14(17 \%)$ \\
Malignancy & $5(6 \%)$ \\
Chronic lung disease & $16(19 \%)$ \\
Chronic renal disease & $5(6 \%)$ \\
Chronic liver disease & $3(4 \%)$ \\
Number of comorbidities & \\
1 & $28(34 \%)$ \\
2 & $17(21 \%)$ \\
3 & $14(17 \%)$ \\
4 & $6(7 \%)$ \\
\hline
\end{tabular}

therapy(CRRT) due to severe renal dysfunction, 1(1.2\%) patients were given extracorporeal membrane oxygenation treatment(ECMO) (Table 6).

\section{Discussion}

This retrospective study described the epidemiological and clinical characteristics of 83 deaths with COVID-19 pneumonia. To my knowledge, our study is the first epidemiological investigation, whose subjects were all patients with COVID-19 pneumonia who died.

In this study, the median of the onset-to-admission interval was longer than that of patients in the previous two studies [9, 14]. Most patients were hospitalized more than 6 days after the onset of the disease, and the longest was 43 days. Two factors likely contributed to the interval. Fisrt, some patients have no severe symptoms in the early stage and it took more for home isolation and community treatment. Second, due to the COVID-19 outbreak, the isolation ward of the hospital may have been under capacity in the initial. Most of the patients who died in the 15-21 days after the onset of the disease, both male and female. This result indicates that the third week may be a period of a high risk of death for critically ill patients with COVID-19.

Most of the deaths with COVID-19 pneumonia were elderly patients especially those over 70 years of age, and male patients. These are consistent with a recent study [9]. The cases of COVID-19 in pregnant women have been mentioned in the previous study [15]. Of 29 pregnant women with COVID-19 in the hospital, there are no deaths so far.

The proportion of patients with comorbidities was higher than previous studies in patients with COVID-19 $[9,14,16]$. The most common comorbidities were 
Table 4 Laboratory findings of 83 deaths with COVID-19 pneumonia on admission

Variables

Blood routine

White blood cell count $\left(\times 10^{9} / \mathrm{L}\right.$; normal range $\left.3.5-9.5\right)$

Decreased

Increased

Neutrophil count $\left(\times 10^{9} / \mathrm{L}\right.$; normal range 1.8-6.3)

Increased

Lymphocyte count $\left(\times 10^{9} / \mathrm{L}\right.$; normal range $\left.1.1-3.2\right)$

Decreased

Mononuclear leucocyte $\left(\times 10^{9} / \mathrm{L}\right.$; normal range $\left.0.1-0.6\right)$

Increased

Hemoglobin (g/L; normal range 130-175 for men; 115-150 for women)

Decreased

Platelets $\left(\times 10^{9} / \mathrm{L}\right.$; normal range $\left.125-350\right)$

Decreased

\section{Blood biochemistry}

Total bilirubin ( $\mu \mathrm{mol} / \mathrm{L}$; normal range $0.0-23.0$ )

Increased

Direct bilirubin ( $\mu \mathrm{mol} / \mathrm{L}$; normal range $0.0-8.0$ )

Increased

Aspartate aminotransferase (U/L; normal range 15.0-40.0)

Increased

Alanine aminotransferase (U/L; normal range 7.0-40.0)

Increased

Alkaline phosphatase (U/L; normal range 50-135)

Increased

Gamma-glutamyl transpeptidas (U/L; normal range 7-45)

Increased

Albumin (g/L; normal range 40-55)

Decreased

Globulin (g/L; normal range 20-40)

Decreased

Increased

Lactate dehydrogenase (U/L; normal range120-250)

Increased

Serum creatinine ( $\mu \mathrm{mol} / \mathrm{L} ;$ normal range 41-81)

Increased

Blood urea nitrogen ( $\mathrm{mmol} / \mathrm{L}$; normal range 3.1-8.8)

Increased

\section{Coagulation function}

Prothrombin time (s; normal range 9.0-13.0)

Increased

Activated partial thromboplastin time (s; normal range 25.0-31.3)

Increased

D-dimer (mg/L; normal range $\leq 0.55$ )
Patients, n(\%)

$9.1(4.7)$

$5(6 \%)$

$34(41 \%)$

6.9(4.4-11.5)

49(59\%)

$0.6(0.4-0.9)$

69(83\%)

$0.4(0.3-0.5)$

16(19\%)

116 (104-121)

$34(41 \%)$

166(72)

24(29\%)

14.1(9.8-19.9)

16(19\%)

5.6(4.2-9.3)

29(35\%)

43(28-62)

47(57\%)

25(19-49)

25(30\%)

76(59-105)

12(14\%)

44(23-75)

$41(49 \%)$

33.7(4.1)

$77(93 \%)$

25.6(22.6-29.0)

$4(5 \%)$

2(2\%)

493(362-682)

79(95\%)

77(55-113)

$39(47 \%)$

9.36(5.50-16.00)

$44(53 \%)$

12.9(12.2-14.2)

$36(43 \%)$

$29.1(27.1-32.5)$

$27(33 \%)$

4.68(1.09-18.00) 
Table 4 Laboratory findings of 83 deaths with COVID-19 pneumonia on admission (Continued)

\begin{tabular}{|c|c|}
\hline Variables & Patients, n(\%) \\
\hline Increased & $78(94 \%)$ \\
\hline \multicolumn{2}{|l|}{ Inflammatory biomarkers } \\
\hline Procalcitonin (ng/mL; normal range $\leq 0.10$ ) & $0.23(0.12-0.94)$ \\
\hline Increased & 69(83\%) \\
\hline C-reactive protein (mg/mL; normal range $\leq 10.0$ ) & 85(47-180.0) \\
\hline Increased & 79(95\%) \\
\hline Interleukin-6 (pg/mL; normal range $\leq 20.0)^{\text {a }}$ & $57.1(38.2-137.6)$ \\
\hline Increased & 19/23(82.6\%) \\
\hline ESR (mm/h; normal range $\leq 15){ }^{\mathbf{b}}$ & $39.75(32.10-63.40)$ \\
\hline Increased & 25/36(69.4\%) \\
\hline \multicolumn{2}{|l|}{ Cardiac biomarkers } \\
\hline Hypersensitive cardiac troponin I $(\mathrm{ng} / \mathrm{mL} \text {; normal range } \leq 0.04)^{c}$ & $0.27(0.09-1.07)$ \\
\hline Increased & $30 / 48(62.5 \%)$ \\
\hline NT-pro B-type natriuretic peptide $(\mathrm{pg} / \mathrm{ml} \text {; normal range } \leq 900)^{\mathbf{d}}$ & $872.0(457.2-1914.5)$ \\
\hline Increased & 19/42(45.2\%) \\
\hline \multicolumn{2}{|l|}{ Blood gas characteristics ${ }^{\mathrm{e}}$} \\
\hline PH (normal range 7.35-7.45) & 7.44(7.39-7.48) \\
\hline $\mathrm{PH}<7.35$ & $13 / 57(22.8 \%)$ \\
\hline $\mathrm{PH}>7.45$ & $24 / 57(42.1 \%)$ \\
\hline $\mathrm{PaO}_{2}(\mathrm{mmHg} ;$ normal range 80-100) & $58.3(43.4-75.9)$ \\
\hline $\mathrm{PaO}_{2}<60 \mathrm{mmHg}$ & $44 / 57$ (77.2\%) \\
\hline $\mathrm{PaCO}_{2}(\mathrm{mmHg}$; normal range 35-45) & $34.1(28.5-37.8)$ \\
\hline $\mathrm{PaO}_{2}<35 \mathrm{mmHg}$ & $33 / 57$ (57.9\%) \\
\hline $\mathrm{PaO}_{2}>50 \mathrm{mmHg}$ & 9/57 (15.8\%) \\
\hline Standard bicarbonate (mmol/L; normal range 21.0-25.0) & $20.5(17.8-24.4)$ \\
\hline
\end{tabular}

Data are mean (SD), median (IQR), and $\mathrm{n}(\%)$. Decreased means over the upper limit of the normal range and increased means below the lower limit of the normal range

${ }^{a}$ Data available for 23 of 83 patients; ${ }^{b}$ Data available for 36 of 83 patients; ${ }^{c}$ Data available for 48 of 83 patients; ${ }^{d}$ Data available for 42 of 83 patients; ${ }^{e}$ Data available for 57 of 83 patients

Table 5 Main lung imaging features on chest $C T$ images of 83 deaths with COVID-19 pneumonia on admission

\begin{tabular}{ll}
\hline & Patients, $\mathbf{n}(\%)$ \\
\hline Uung imaging features & \\
Unilateral pneumonia & $12(14.5 \%)$ \\
Bilateral pneumonia & $71(85.5 \%)$ \\
Ground-glass opacity & $83(100 \%)$ \\
Consolidation & $33(39.8 \%)$ \\
Air bronchogram & $18(21.7 \%)$ \\
Bronchial dilatation & $23(27.7 \%)$ \\
Pleural effusion or thickening & $47(56.6 \%)$ \\
\hline
\end{tabular}

hypertension in our study, which was diabetes in two previous cohort studies of Middle Eastern respiratory syndrome coronavirus (MRSE-Cov) infection, and severe acute respiratory syndrome coronavirus(SARS-CoV) infection $[17,18]$. We observed that the majority of patients who died were also geriatric patients and those suffering from chronic comorbidities. However, some healthy people(22\%) died without complications, which were an indication of the high pathogenicity of COVID19. The initial clinical symptoms of patients infected with COVID-19 were nonspecific. There were no significant differences in the types of initial symptoms between the deaths in our study and the recently published studies $[9,14,16,19]$. However, the first three symptoms in our study were shortness of breath, fever, myalgia, or fatigue. A small number of patients initially presented with gastrointestinal symptoms, such as anorexia, nausea, vomiting, and diarrhea, which were mentioned in previous studies $[9,16]$. 


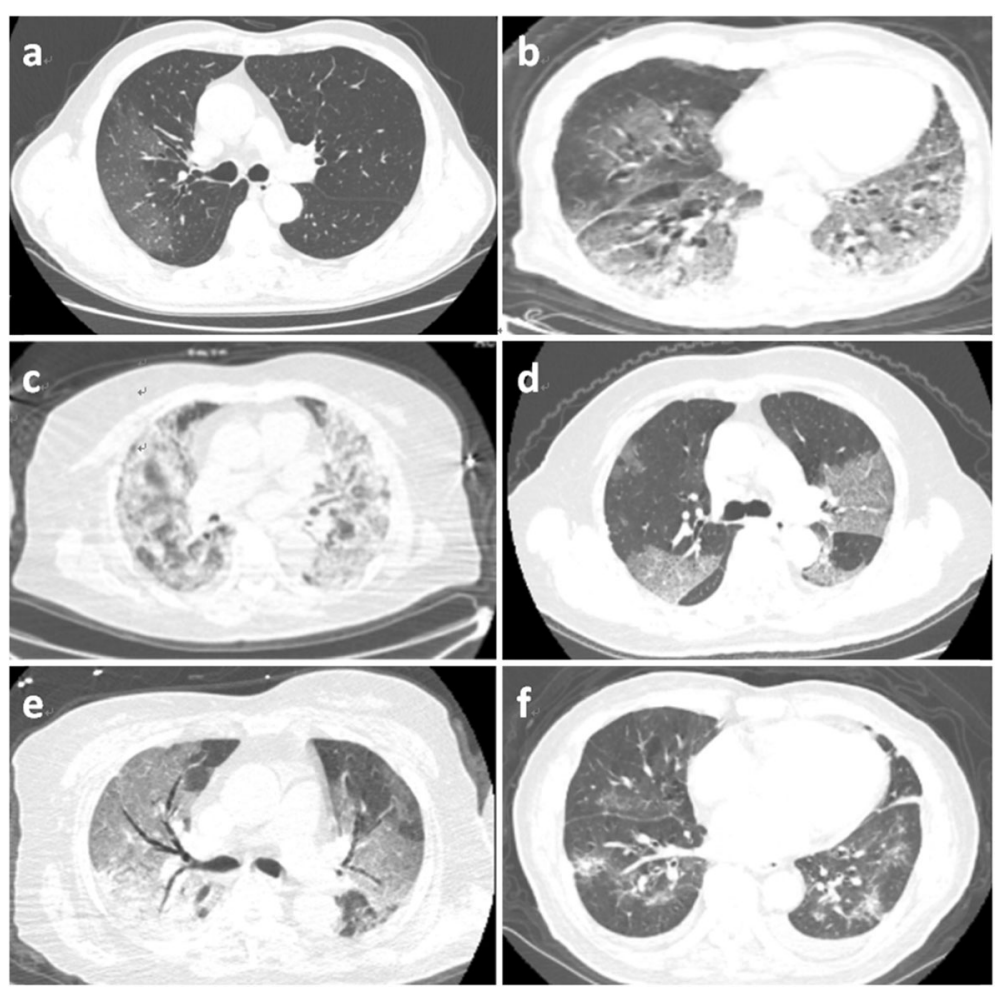

Fig. 2 Lung imaging features of axial chest CT scans. a Ground glass opacity(GGO) on the right lung in a patient between 60 and 69 years of age; b Multiple GGO and bronchial dilatation of both lungs in a patient between 60 and 69 years of age; c Multiple GGO and consolidation in a patient between 60 and 69 years of age; d Multiple GGO of both lungs and local consolidation in a patient between 70 and 79 years of age; e Multiple GGO and air bronchogram in a patient between 60 and 69 years of age; $\mathbf{f}$ Multiple GGO and pleural effusion in a patient between 80 and 89 years of age

Most of the patients in our study developed acute respiratory failure(ARF) including ARDS, fatal infection, abnormal coagulation, and eventually multiple organ failure, except for six who died of acute myocardial infarction. $12(14.5 \%)$ patients with unilateral pneumonia at admission were dead in our study. We analyzed the causes of death of these patients are as follows: First, the patient could progress to bilateral lung infection and even severe ARF after admission. Secondly, some patients have severe comorbidities such as chronic heart failure, chronic lung disease, chronic renal disease. Finally, some patients develop serious complications such as acute myocardial infarction, multiple organ failure.

As a newly identified disease, little is known about the pathogenic mechanism of COVID-19. Most of the patients who died had abnormal coagulation. Increased inflammatory markers such as procalcitonin and Creactive, lymphopenia were a common characteristic in the patients. This series of changes is a manifestation of the immune response and maybe be a factor in poor prognosis $[9,14,18]$. In a recent fatal case report, typical features of inflammation were observed in the pulmonary pathology of the patient, whose pathological section showed interstitial mononuclear inflammatory infiltrates, dominated by lymphocytes [20]. These pathological characteristics greatly resemble those of MRSE-Cov infection and SARS-CoV infection [20-22].

Until now, no drugs are specifically effective against coronaviruses. Of the 83 patients in our study, each patient received Abidol, and some patients were treated with Oseltamivir, Ganciclovir, Lopinavir, and Ritonavir, but none of them had a definite therapeutic effect. Besides, Radcivir is an unlisted nucleotide drug whose broad-spectrum antiviral activity has been confirmed in animal models [23, 24]. It may be a potentially effective drug for patients with COVID-19 [20]. Two randomized controlled clinical trials (NCT04252664; NCT04257656) to assess the safety and efficacy of Radcivir are is currently underway in patients hospitalized with COVID-19 pneumonia.

The patients in this study were generally had associated with a secondary bacterial infection, followed by sepsis and septic shock. $97.6 \%$ of the patients were treated with antibiotics based on abnormal inflammatory markers and bacterial culture results.

In patients with SARS and MERS, the effect of glucocorticoid therapy on prognosis is controversial $[25,26]$. However, severe patients with COVID-19 may be 
Table 6 Bacterial culture results, complications, and treatment of 83 deaths with COVID-19 pneumonia after admission

\begin{tabular}{|c|c|}
\hline & Patients, $\mathrm{n}(\%)$ \\
\hline Bacterial culture results & $36(43.4 \%)$ \\
\hline Sputum bacteria culture positive & $22(26.5 \%)$ \\
\hline Blood bacteria culture positive & $9(10.8 \%)$ \\
\hline Urine bacteria culture positive & $5(6.2 \%)$ \\
\hline Fecal bacteria culture positive & 0 \\
\hline \multicolumn{2}{|l|}{ Complications } \\
\hline Acute respiratory failure & $71(85.5 \%)$ \\
\hline Acute myocardial infarction & $6(7.2 \%)$ \\
\hline Heart failure & $37(44.6 \%)$ \\
\hline Acute liver injury & $9(10.8 \%)$ \\
\hline Acute kidney injury & $22(26.5 \%)$ \\
\hline Gastrointestinal bleeding & 16(19.2\%) \\
\hline Sepsis & $69(83.1 \%)$ \\
\hline Shock & $33(39.8 \%)$ \\
\hline \multicolumn{2}{|l|}{ Treatments } \\
\hline Antiviral therapy & $83(100 \%)$ \\
\hline \multicolumn{2}{|l|}{ Monotherapy } \\
\hline Abidole & $6(7.2 \%)$ \\
\hline \multicolumn{2}{|l|}{ Combination therapyAbidole } \\
\hline Abidole/ Oseltamivir & $57(68.7 \%)$ \\
\hline Abidole/ Ribavirin & $16(19.3 \%)$ \\
\hline Abidole/ Lopinavir and Ritonavir & $3(3.6 \%)$ \\
\hline Abidole/ Oseltamivir/ Lopinavir and Ritonavir & $1(1.2 \% \%)$ \\
\hline Antifungal therapy & $2(2.4 \% \%)$ \\
\hline Antibiotic therapy & $81(97.6 \%)$ \\
\hline Penicillins & $24(28.9 \%)$ \\
\hline Cephalosporins & $52(62.7 \%)$ \\
\hline Quinolone & $35(42.2 \%)$ \\
\hline Vancomycin & $11(13.3 \%)$ \\
\hline Glucocorticoid therapy & $54(65.1 \%)$ \\
\hline Intravenous immunoglobulin therapy & $39(47.0 \%)$ \\
\hline CRRT & $6(7.3 \%)$ \\
\hline Invasive mechanical ventilation & $20(24.1 \%)$ \\
\hline Non-invasive mechanical ventilation & $51(61.4 \%)$ \\
\hline High flow nasal cannula & $12(14.5 \%)$ \\
\hline ECMO & $1(1.2 \%)$ \\
\hline
\end{tabular}

CRRT continuous renal replacement therapy, ECMO extracorporeal membrane oxygenation

beneficial from glucocorticoid therapy to prevent ARDS development, based on recent studies [16, 20]. $65.1 \%$ of the patients in this study received glucocorticoid therapy.

Critical illness among patients hospitalized with COVID-19 is common and associated with a high frequency of IMV in some recent large sample studies [10, $27,28]$. In our study, 63 patients did not receive IMV, 41 of whom declined IMV. Of the 41 patients who declined
IMV, 9 patients declined IMV themselves, and 32 patients were completely incapacitated due to serious illness and the family members declined it to relieve the suffering of the patient considering the patient's advanced age and poor prognosis. The other 22 patients did not receive IMV for unknown reasons lacking medical records, which did not rule out a medical run in the early stages of the COVID-19 outbreak. The timing of intubation in patients with severe COVID-19 pneumonia is challenging. Most patients with acute respiratory distress syndrome (ARDS) due to COVID-19 will warrant intubation and mechanical ventilation $[9,10,28]$. Delaying IMV until the patient acutely decompensates is potentially harmful to the patient and affects prognosis [29].

Our study has several limitations. First of all, the study had a limited number of cases, with only 83 deaths. However, to our knowledge, very few case series of deaths have been reported, the data is a valuable demonstration of characteristics of deaths with COVID-19 pneumonia in the early period of exponential growth. Secondly, some data such as cytokines (eg, IL2, IL4, IL6, IL10, TNF, IFN $\gamma$ ) were absent in patients admitted early, which were related to lung injury in previous studies SARS-CoV and MERS-CoV [30, 31]. We will routinely observe the changes of cytokines of patients in further study. Thirdly, the study has not compared the difference between surviving and dead patients due to the initial design. However, this is a series of study designs and the patients will continue to be followed up.

\section{Conclusions}

This single-center retrospective case series early shows the epidemiological and clinical characteristics of deaths with COVID-19 pneumonia. Most of the deaths with COVID19 pneumonia were elderly patients with underlying comorbid diseases, especially those over 70 years of age. The time of death was mostly 15-21 days after the onset of the disease. More care should be given to the elderly in further prevention and control strategies of COVID-19.

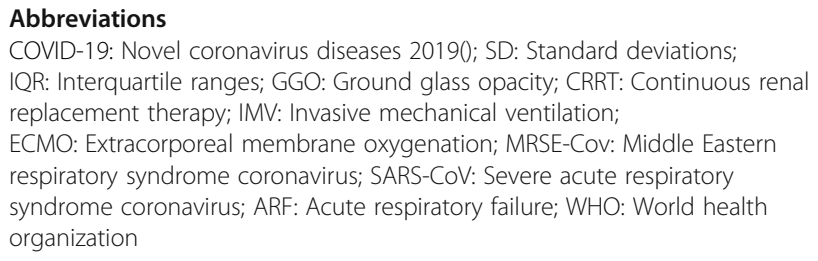

\section{Acknowledgments}

We thank all patients and their families involved in the study.

\section{Authors' contributions}

$T Y, Y G$, and QC contributed equally to this article. TY, ZCL and ZHZ conceived and designed the study. YG, QC, BP, YC, JSL, CPH, CH, JP, JJW, YQZ, JY, and JHT collected the data, analyzed, and interpreted data. YG and QC performed the statistical analysis. BP coordinated the research groups. TY were the main contributors to writing the first draft. YG and QC revised the final manuscript. All authors read and approved the final manuscript. 


\section{Funding}

No funding.

\section{Availability of data and materials}

The data will be available from the corresponding author on a reasonable request. After the publication of this study, the participant data without names an identifiers will be made available after approval from the corresponding authors and Wuhan University Renmin Hospital.

\section{Ethics approval and consent to participate}

This study protocol complied with the Medical Ethical Committee of Wuhan University Renmin Hospital (No.WDYR2020-k050). Written informed consent was waived due to the rapid emergence of this infectious disease with the permission of the Medical Ethical Committee.

\section{Consent for publication}

Not applicable.

\section{Competing interests}

We declare that we have no conflicts of interest.

\section{Author details}

'Union Department of Infection Disease, Wuhan University Renmin Hospital, Wuhan 430060, China. ${ }^{2}$ Department of Neurology, Wuhan University Renmin Hospital, Wuhan 430060, China. ${ }^{3}$ Department of Gastroenterology, Wuhan University Renmin Hospital, Wuhan 430060, China. ${ }^{4}$ Department of Critical Care Medicine, Wuhan University Renmin Hospital, Wuhan 430060, China. ${ }^{5}$ Department of Obstetrics and Gynecology, Wuhan University Renmin Hospital, Wuhan 430060, China.

Received: 16 March 2020 Accepted: 15 September 2020

Published online: 22 September 2020

\section{References}

1. Avramovski P, Avramovska M, Sikole A. Bone strength and arterial stiffness impact on cardiovascular mortality in a general population. J Osteoporos. 2016;2016:7030272.

2. Lu H, Stratton CW, Tang Y-W. Outbreak of pneumonia of unknown etiology in Wuhan, China: the mystery and the miracle. J Med Virol. 2020:92(4):401-2.

3. Wang D, Hu B, Hu C, Zhu F, Liu X, Zhang J, et al. Clinical characteristics of 138 hospitalized patients with 2019 novel coronavirus-infected pneumonia in Wuhan. China JAMA. 2020:323(11):1061-9.

4. WHO. Coronavirus disease 2019 (COVID-19) Situation Report -97. https:// www.who.int/docs/default-source/coronaviruse/situation-reports/20200426sitrep-97-covid-19.pdf Accessed 1 July 2020.

5. Jernigan DB. Update: public health response to the coronavirus disease 2019 outbreak - United States, February 24, 2020. MMWR Morb Mortal Wkly Rep. 2020;69(8):216-9.

6. Day M. Covid-19: Italy confirms 11 deaths as cases spread from north. BMJ (Clinical research ed). 2020;368:m757.

7. Deng SQ, Peng HJ. Characteristics of and Public Health Responses to the Coronavirus Disease 2019 Outbreak in China. J Clin Med. 2020;9(2):575. https://doi.org/10.3390/jcm9020575.

8. WHO. WHO Coronavirus Disease (COVID-19) Dashboard. https://covid19. who.int/ Accessed 1 July 2020

9. Yang $X, Y u Y, X u$ J, Shu H, Xia J, Liu H, et al. Clinical course and outcomes of critically ill patients with SARS-CoV-2 pneumonia in Wuhan, China: a singlecentered, retrospective, observational study. Lancet Respir Med. 2020;8(5):475-81.

10. Cummings MJ, Baldwin MR, Abrams D, Jacobson SD, Meyer BJ, Balough EM, et al. Epidemiology, clinical course, and outcomes of critically ill adults with COVID-19 in New York City: a prospective cohort study. Lancet (London, England). 2020;395(10239):1763-70

11. Chen T, Wu D, Chen H, Yan W, Yang D, Chen G, et al. Clinical characteristics of 113 deceased patients with coronavirus disease 2019: retrospective study. BMJ (Clinical research ed). 2020;368:m1091.

12. National Health Commission of the People's Republic of China. New coronavirus pneumonia prevention and control program (4th edn). 2020. http://www.gov.cn/zhengce/zhengceku/2020-01/28/5472673/files/0f96c1 0cc09d4d36a6f9a9fob42d972b.pdf Accessed 1 July 2020.
13. WHO. Laboratory testing for 2019 novel coronavirus (2019-nCoV) in suspected human cases. 2020. https:/wwww.who.int/publications-detail/laboratory-testing-for-2 019-novel-coronavirus-in-suspected-human-cases-20200117 Accessed 1 July 2020.

14. Huang C, Wang Y, Li X, Ren L, Zhao J, Hu Y, et al. Clinical features of patients infected with 2019 novel coronavirus in Wuhan, China. Lancet (London, England). 2020;395(10223):497-506.

15. Chen H, Guo J, Wang C, Luo F, Yu X, Zhang W, et al. Clinical characteristics and intrauterine vertical transmission potential of COVD-19 infection in nine pregnant women: a retrospective review of medical records. Lancet. 2020;395(10226):809-15.

16. Chen N, Zhou M, Dong X, Qu J, Gong F, Han Y, et al. Epidemiological and clinical characteristics of 99 cases of 2019 novel coronavirus pneumonia in Wuhan, China: a descriptive study. Lancet (London, England). 2020;395(10223):507-13.

17. Lew TWK, Kwek T-K, Tai D, Earnest A, Loo S, Singh K, et al. Acute respiratory distress syndrome in critically ill patients with severe acute respiratory syndrome. JAMA. 2003;290(3):374-80.

18. Arabi YM, Arifi AA, Balkhy HH, Najm H, Aldawood AS, Ghabashi A, et al. Clinical course and outcomes of critically ill patients with Middle East respiratory syndrome coronavirus infection. Ann Intern Med. 2014;160(6):389-97.

19. Wu Y, Xu X, Chen Z, Duan J, Hashimoto K, Yang L, et al. Nervous system involvement after infection with COVID-19 and other coronaviruses. Brain Behav Immun. 2020

20. Wang M, Cao R, Zhang L, Yang X, Liu J, Xu M, et al. Remdesivir and chloroquine effectively inhibit the recently emerged novel coronavirus (2019-nCoV in vitro. Cell Res. 2020.

21. Ng DL, Al Hosani F, Keating MK, Gerber SI, Jones TL, Metcalfe MG, et al. Clinicopathologic, Immunohistochemical, and Ultrastructural findings of a fatal case of Middle East respiratory syndrome coronavirus infection in the United Arab Emirates, April 2014. Am J Pathol. 2016;186(3):652-8.

22. Ding Y, Wang H, Shen H, Li Z, Geng J, Han H, et al. The clinical pathology of severe acute respiratory syndrome (SARS): a report from China. J Pathol. 2003;200(3):282-9.

23. de Wit E, Feldmann F, Cronin J, et al. Prophylactic and therapeutic remdesivir (GS5734) treatment in the rhesus macaque model of MERS-CoV infection. Proc Natl Acad Sci U S A. 2020;117(12):6771-6. https://doi.org/10.1073/pnas.1922083117.

24. Sheahan TP, Sims AC, Leist SR, Schäfer A, Won J, Brown AJ, et al. Comparative therapeutic efficacy of remdesivir and combination lopinavir, ritonavir, and interferon beta against MERS-CoV. Nat Commun. 2020;11(1):222.

25. Arabi YM, Mandourah Y, Al-Hameed F, Sindi AA, Almekhlafi GA, Hussein MA, et al. Corticosteroid therapy for critically ill patients with Middle East respiratory syndrome. Am J Respir Crit Care Med. 2018;197(6):757-67.

26. Stockman LJ, Bellamy R, Garner P. SARS: systematic review of treatment effects. PLoS Med. 2006;3(9):e343-e.

27. Richardson S, Hirsch JS, Narasimhan M, Crawford JM, McGinn T, Davidson KW, et al. Presenting characteristics, comorbidities, and outcomes among 5700 patients hospitalized with COVID-19 in the New York City area. Jama. 2020;323(20):2052-9.

28. Grasselli G, Zangrillo A, Zanella A, Antonelli M, Cabrini L, Castelli A, et al. Baseline characteristics and outcomes of 1591 patients infected with SARS-CoV-2 admitted to ICUs of the Lombardy region. Italy JAMA. 2020;323(16):1574-81.

29. Anesi GL. Coronavirus disease 2019 (COVID-19): critical care and airway management issues. UpToDate 2020. https://www.uptodate.com/contents/ coronavirus-disease-2019-covid-19-critical-care-and-airway-managementissues Accessed 1 July 2020.

30. Mahallawi WH, Khabour OF, Zhang Q, Makhdoum HM, Suliman BA. MERSCoV infection in humans is associated with a pro-inflammatory Th1 and Th17 cytokine profile. Cytokine. 2018;104:8-13.

31. Wong CK, Lam CW, Wu AK, Ip WK, Lee NL, Chan IH, et al. Plasma inflammatory cytokines and chemokines in severe acute respiratory syndrome. Clin Exp Immunol. 2004;136(1):95-103.

\section{Publisher's Note}

Springer Nature remains neutral with regard to jurisdictional claims in published maps and institutional affiliations. 